\title{
Changes in HER2 Expression in Breast Cancer Xenografts After Therapy Can Be Quantified Using PET and ${ }^{18}$ F-Labeled Affibody Molecules
}

\author{
Gabriela Kramer-Marek ${ }^{1}$, Dale O. Kiesewetter ${ }^{2}$, and Jacek Capala ${ }^{1}$ \\ ${ }^{I}$ National Cancer Institute, National Institutes of Health, Bethesda, Maryland; and ${ }^{2}$ National Institute of Biomedical Imaging and \\ Bioengineering, National Institutes of Health, Bethesda, Maryland
}

In vivo imaging of human epidermal growth factor receptor type 2 (HER2) expression may allow direct assessment of HER2 status in tumor tissue and provide a means to quantify changes in receptor expression after HER2-targeted therapies. This work describes the in vivo characterization of the HER2-specific $N-2-\left(4-{ }^{18} \mathrm{~F}\right.$-fluorobenzamido)ethyl]maleimide $\left({ }^{18} \mathrm{~F}-\mathrm{FBEM}\right)-\mathrm{Z}_{\text {HER2:342 }}$ Affibody molecule and its application to study the effect of 17 (dimethylaminoethylamino)-17-demethoxygeldanamycin (17-DMAG) on HER2 expression by PET. Methods: To assess the correlation of signal observed by PET with receptor expression, we administered the tracer to athymic nude mice bearing subcutaneous human breast cancer xenografts with different levels of HER2 expression. To study the downregulation of HER2, we treated the mice with 4 doses (40 mg/kg) of 17-DMAG, an inhibitor of heat-shock protein 90, known to decrease HER2 expression. The animals were scanned before and after treatment. After the last scan, the mice were euthanized and tumors were frozen for receptor analysis. Results: The tracer was eliminated quickly from the blood and normal tissues, providing high tumor-to-blood and tumor-to-muscle ratios as early as $20 \mathrm{~min}$ after injection. The high-contrast images between normal and tumor tissue were recorded for BT474 and MCF7/ clone18 tumors. Low but still detectable uptake was observed for MCF7 tumors, and none for MDA-MB-468. The signal correlated with the receptor expression as assessed by immunohistochemistry, Western blot, and enzyme-linked immunosorbent assay. The levels of HER2 expression estimated by post-treatment PET decreased $71 \%\left(P<4 \times 10^{-6}\right)$ and $33 \%(P<0.002)$, respectively, for mice bearing BT474 and MCF7/clone18 tumors. These changes were confirmed by the biodistribution studies, enzyme-linked immunosorbent assay, and Western blot. Conclusion: Our results suggest that the described ${ }^{18} \mathrm{~F}-\mathrm{FBEM}-\mathrm{Z}_{\text {HER2:342 }}$ Affibody molecule can be used to assess HER2 expression in vivo by PET and monitor possible changes of receptor expression in response to therapeutic interventions.

Key Words: Affibody molecule; molecular imaging; HER2; breast cancer; PET

J Nucl Med 2009; 50:1131-1139

DOI: 10.2967/jnumed.108.057695

Received Sep. 1, 2008; revision accepted Dec. 19, 2008.

For correspondence or reprints contact: Jacek Capala, 10 Center Dr.,

Bldg. 10, Room 1B-37A, Bethesda, MD 20892.

E-mail: capalaj@mail.nih.gov

COPYRIGHT () 2009 by the Society of Nuclear Medicine, Inc.
H uman epidermal growth factor receptor type 2 (HER2, ErbB2, neu) is a well-established tumor biomarker that is overexpressed in a wide variety of carcinomas including breast, ovary, prostate, and lung cancer $(1,2)$. Because HER2 overexpression plays an important role in aggressive tumor behavior and poor clinical outcome (3), early-stage detection and quantification of HER2 is clinically relevant and can be used for selection of optimal therapy for individual patients.

Heat-shock protein 90 (Hsp90) is a molecular chaperone highly expressed in most tumor cells. It is required for the stability and function of many client proteins that promote cancer cell growth and survival (4). Hsp90 inhibitors, by interfering with the chaperone activity, result in targeting of client proteins to the proteasome for degradation. Because HER2 is one of the Hsp90 client proteins, it can be indirectly downregulated by Hsp90 inhibitors such as the naturally occurring ansamycin antibiotic geldanamycin known for its tumoricidal potential (5) or the recently developed 17(dimethylaminoethylamino)-17-demethoxygeldanamycin (17-DMAG), which is a hydrophilic geldanamycin derivative that can be administered orally with good bioavailability and better activity in vitro and in vivo than its predecessor, 17(allylamino)-17-demethoxygeldanamycin (17-AAG) (6). In vivo downregulation of HER2 in human tumor xenografts after treatment with 17-AAG was reported by Smith-Jones et al. (7) and, later, by Orlova et al. (8). These geldanamycin analogs are currently being tested in phase I clinical trials (9).

Thus far, the HER2 expression pattern has been routinely determined by ex vivo analysis of tissue samples using fluorescence in situ hybridization or immunohistochemistry. These methods, although commonly used in clinical practice, have several limitations. Most notably, they require tissue removal from the body, which restricts their analysis only to the sampled parts and may not properly represent the overall tumor characteristics. Variability in scoring between these 
techniques, whether as a result of true heterogeneity or artifacts in preparation, has led to decreased reliability of the final HER2 status determination $(10,11)$.

Introducing a new methodology for in vivo quantification of HER2 receptors using PET would present a complementary, noninvasive option to obtain real-time information that not only could facilitate selection of patients for HER2targeted therapy $(3,12)$ but also could provide information on the immediate response to therapeutic interventions, both in primary lesions and in distant metastases. This feedback would allow adjustment of the dose and treatment schedule for individual patients, based on the actual status of the HER2 receptors. PET, with its high sensitivity, high spatial resolution, and proven quantification abilities, could also reduce the number of false-negative or false-positive results from the currently used methods: fluorescence in situ hybridization and immunohistochemistry.

Several molecular probes based on antibodies have recently been tested in experimental animal tumor models, but a PET tracer for routine clinical use has not yet been developed. The clinical application of antibodies $(\sim 150$ $\mathrm{kDa}$ ) to molecular imaging is limited because of their large size, resulting in low tumor penetration and slow clearance. Often, several days are needed to obtain reasonable tumorto-blood ratios, making most short-lived PET radionuclides inapplicable. Several different ligands have been developed and extensively studied over the last few years to circumvent these problems. Among these ligands are antibody fragments and engineered variants such as $\mathrm{F}\left(\mathrm{ab}^{\prime}\right)_{2}, \mathrm{~F}\left(\mathrm{ab}^{\prime}\right)$, single-chain Fv, diabodies, and minibodies (13).

Recently, a new class of relatively small $(\sim 6.5 \mathrm{kDa})$ proteins has become available for studies, and several groups are now using Affibody molecules to image HER2 $(14,15)$ or epidermal growth factor receptor-positive tumors (16). The small size, resulting in rapid blood clearance, good tumor penetration, and high binding affinity to selected targets, makes Affibody molecules ideal candidates for imaging purposes. HER2-specific Affibody molecules, which bind with picomolar affinity to a HER2 epitope distinct from those involved in binding of trastuzumab or pertuzumab, have been labeled with several radioisotopes, including ${ }^{99 \mathrm{~m}} \mathrm{Tc},{ }^{111} \mathrm{In},{ }^{68} \mathrm{Ga},{ }^{90} \mathrm{Y}$, and ${ }^{125} \mathrm{I}$ (8,17-19). A methodology for labeling Affibody molecules with ${ }^{18} \mathrm{~F}$ has recently been developed in our laboratory $(14,20)$. We have reported great potential for $N-2-\left(4-{ }^{18} \mathrm{~F}-\right.$ fluorobenzamido)ethyl]maleimide $\quad\left({ }^{18} \mathrm{~F}-\mathrm{FBEM}\right)-\mathrm{Z}_{\text {HER2:342 }}$ Affibody molecules in the in vivo monitoring of HER2 expression by PET. Cheng et al. (15) used a similar approach to test various clones of anti-HER2 Affibody molecules and obtained comparable results.

In the current study, we tested the hypothesis that the use of ${ }^{18}$ F-FBEM-Z $Z_{H E R 2: 342}$ Affibody molecules would enable quantitative assessment of HER2 downregulation after antiHER2 therapy. To our knowledge, we are the first to show that semiquantitative analysis of PET data can be used to assess different levels of HER2 expression and to monitor changes in expression in response to 17-DMAG treatment in mice bearing xenograft tumors.

\section{MATERIALS AND METHODS}

\section{General}

Unless otherwise specified, all reagents were of analytic grade and were obtained from commercial sources. The $\mathrm{Z}_{\text {HER2:342-Cys }}$ Affibody molecules were kindly provided by our Cooperative Research and Development Agreement partner in Sweden (Affibody $\mathrm{AB}) .{ }^{18} \mathrm{~F}$ radionuclide was produced via the ${ }^{18} \mathrm{O}(\mathrm{p}, \mathrm{n}){ }^{18} \mathrm{~F}$ nuclear reaction in the Clinical Center of the National Institutes of Health cyclotron facility by irradiating ${ }^{18} \mathrm{O}$-enriched water. ${ }^{18} \mathrm{~F}$ FBEM-Z $\mathrm{Z}_{\text {HER2:342 }}$ Affibody was prepared as previously described (20). 17-DMAG was purchased from InvivoGen as lyophilized purple powder and reconstituted with $0.9 \%$ sodium chloride for injection.

\section{Cell Lines}

Human breast (BT474, MDA-MB-361, MCF7, and MDA-MB468) cancer cell lines that express different levels of $H E R 2$ were purchased from the American Type Culture Collection. The breast cancer cell line stably transfected with HER2 (MCF7/clone18) was kindly provided by Drs. John W. Park and Byron Hann, University of California, San Francisco. The cells were cultured in RPMI 1640 medium (BT474 and MDA-MB-468) and in Dulbecco modified Eagle medium (MCF7, MDA-MB-361, and MCF7/ clone18) supplemented with $10 \%(\mathrm{v} / \mathrm{v})$ heat-inactivated fetal bovine serum (GIBCO) and penicillin/streptomycin (a $100 \mathrm{U} /$ $\mathrm{mL}$ concentration of each). Cells were grown as a monolayer at $37^{\circ} \mathrm{C}$ in a humidified atmosphere containing $5 \% \mathrm{CO}_{2}$. In the case of MCF7/clone18 transfectants, the culture medium also contained $400 \mu \mathrm{g}$ of Geneticin (GIBCO) per milliliter.

\section{Tumor Model}

All animal studies were conducted in accordance with the principles and procedures outlined in the Guide for the Care and Use of Laboratory Animals (21) and were approved by the Institutional Animal Care and Use Committee of the National Institutes of Health. Cells suspended in Matrigel (BD Bioscience) were implanted subcutaneously into the shoulder region of 5- to 7-wk-old female athymic nude mice: $4.5 \times 10^{6}$ MDA-MB-361, MCF7, MDA-MB-468, or MCF7/clone18 cells or $5.5 \times 10^{6}$ BT474 cells. Estrogen pellets $(1.72 \mathrm{mg}$; Innovative Research of America) were implanted $48 \mathrm{~h}$ before tumor cell inoculation and remained in place until the end of the study. Tumors (100-250 mg) developed after 3-5 wk. The mice were treated with 4 doses (intravenous, $40 \mathrm{mg} / \mathrm{kg}$ ) of 17-DMAG, an inhibitor of Hsp90, to monitor the downregulation of HER2 receptors. The animals were dosed at 12, 36, 48, and $60 \mathrm{~h}$ after the first PET scan and then were imaged again before $72 \mathrm{~h}$.

\section{Biodistribution Studies}

${ }^{18}$ F-FBEM-Z $Z_{H E R 2: 342}$ Affibody (3.7-4.4 MBq, 3.4-4.0 $\mu \mathrm{g}, 100$ $\mu \mathrm{L})$ was injected into the tail vein of mice bearing BT474 and MCF7/clone18 tumors. Groups of 6-7 mice were sacrificed, and their major organs were dissected and weighed $2 \mathrm{~h}$ after injection. The radioactivity uptake in the tissues was measured, along with a standard of the injected dose, using a $\gamma$-counter. The results were expressed as percentage injected dose (\%ID) per gram of tissue. 


\section{PET Studies}

The mice were anesthetized using isoflurane $/ \mathrm{O}_{2}(1.5 \%-5 \% \mathrm{v} / \mathrm{v})$, placed prone in the center of the field of view of the scanner, and given an injection of ${ }^{18} \mathrm{~F}-\mathrm{FBEM}-\mathrm{Z}_{\text {HER } 2: 342}$ Affibody (3.7-4.4 MBq, 3.4-4.0 $\mu \mathrm{g}, 100 \mu \mathrm{L})$ via the tail vein. PET scans were performed using the Advanced Technology Laboratory Animal Scanner (ATLAS) (22). Whole-body (4 bed positions, each $15 \mathrm{~min}$ ) or dynamic data acquisition (6 or 12 frames, 10 min per frame) was started about $2 \mathrm{~min}$ after radiotracer injection and was recorded with a 100- to 700-keV energy window. In some cases, additional static scans (15 min) were obtained $1 \mathrm{~h}$ after tracer injection. The choice of time points was based on previous studies in which uptake maximized at $1 \mathrm{~h}$ after injection and then maintained a plateau until $2 \mathrm{~h}$ (14). The images were reconstructed by a 2-dimensional ordered-subsets expectation maximum algorithm, and no correction was applied for attenuation or scatter. For each scan, regions of interest were drawn over the tumor, normal tissue, and major organs. The maximum counts per pixel within the tumor or organs were obtained from the multiple regions of interest (counts $/ \mathrm{s} / \mathrm{cm}^{3}$ ). The results were calculated as $\% \mathrm{ID} / \mathrm{g}$ by means of a calibration constant obtained from scanning the ${ }^{18} \mathrm{~F}$ source of known activity, assuming a tissue density of $1 \mathrm{~g} / \mathrm{mL}$, and dividing by the injected dose, decay-corrected to the time of scanning.

\section{Fluorescence-Activated Cell Sorter (FACS) Analysis}

Subconfluent cell cultures were incubated with $50 \mu \mathrm{g}$ of Affibody molecules per microliter or just with growth medium before fixation for $1 \mathrm{~h}$ at $37^{\circ} \mathrm{C}$. Then, the cells were washed twice with phosphate-buffered saline (PBS ${ }^{-}$) and detached with Cellstripper (Cellgro). For every sample, $1 \times 10^{6}$ cells were collected, fixed with $4 \%$ formaldehyde, and incubated for $1 \mathrm{~h}$ on ice. The cells were then washed 3 times with $\mathrm{PBS}^{-}$and resuspended in stain buffer PBS $^{-}$plus 5\% fetal bovine serum and $0.5 \%$ Triton X-100 [Union Carbide]). Subsequently, the cells were covered and incubated on ice for $30 \mathrm{~min}$, and afterward the appropriate dilution of goat-anti-Affibody antibody was added for $45 \mathrm{~min}$ to cells treated beforehand with Affibody molecules. The cells were then washed once and resuspended in $200 \mu \mathrm{L}$ of stain buffer, followed by a 30-min incubation with secondary rabbit-antigoat-AlexaFluor488 conjugated antibody (Molecular Probe). For HER2 detection, cells, after permeabilization, were incubated for 45 min with antimouse $\mathrm{Neu}(24 \mathrm{D} 2)$-fluorescein isothiocyanate monoclonal antibody or with $\mathrm{IgG}_{1}$-fluorescein isothiocyanate mouse isotypic control (Santa Cruz Biotechnology, Inc.).

After antibody staining, all cells were washed with stain buffer 2 times and resuspended in $300 \mu \mathrm{L}$ of $\mathrm{PBS}^{-}$for data acquisition. All solutions were kept on ice.

Flow cytometry was done using a FACS Calibur instrument (BD Biosciences). CellQuest Pro software was used for data acquisition and FlowJo software for analysis (Tree Star Inc.). For each sample, 10,000 events were recorded, and the population corresponding to single cells was gated and analyzed as a histogram plot.

\section{Enzyme-Linked Immunosorbent Assay (ELISA)}

Cell Lysates. The cell cultures were harvested by Cellstripper and washed 3 times in PBS to determine the HER2 protein level in cell lines. Then, the cell pellets were resuspended in a proper amount of resuspension buffer provided with the ELISA kit (Calbiochem). For each $100 \mu \mathrm{L}$ of cell suspension, $20 \mu \mathrm{L}$ of antigen extraction agent (also provided with the kit) were added, and the cells were incubated at $4{ }^{\circ} \mathrm{C}$ for $20 \mathrm{~min}$ with gentle agitation and then centrifuged at $13,000 \mathrm{rpm}$ for $15 \mathrm{~min}$. The supernatant was collected and stored at $-80^{\circ} \mathrm{C}$.

Preparation of Tumor Tissue. The mice were sacrificed by cervical dislocation, and the tumors were isolated, frozen on dry ice, and stored at $-80^{\circ} \mathrm{C}$. Afterward, the specimens were weighed and sliced into small pieces. Then, tumor tissue was homogenized on ice, using a Polytron homogenizer, and lysed in resuspension buffer provided with the ELISA kit. Cell debris was removed through centrifugation of the suspensions at $13,000 \mathrm{rpm}\left(4^{\circ} \mathrm{C}\right)$ for $15 \mathrm{~min}$. The supernatants were collected and stored at $-80^{\circ} \mathrm{C}$. Before use, the protein concentration was estimated by the bicinchoninic acid assay kit (Pierce), according to the manufacturer's protocol. The HER2 protein level was measured by an ELISA kit, according to the manufacturer's recommended procedure. Results are expressed in nanograms of HER 2 per milligram of protein. The data are presented as the mean of 2 replicates, with each experiment repeated 3 times.

\section{Western Blot Analysis}

All reagents for NuPAGE and Western blots were from Invitrogen. Total cellular protein (30 $\mu \mathrm{g}$ for all analyses) was mixed with sample buffer and incubated at $70^{\circ} \mathrm{C}$ for $10 \mathrm{~min}$. Thirty-microliter aliquots were resolved on $4 \%-12 \%$ NuPAGE Novex tris-acetate gel by electrophoresis. Thereafter, proteins were transferred to a nitrocellulose membrane, which was subsequently blocked for $1 \mathrm{~h}$ at room temperature with $5 \%$ nonfat milk blocking buffer and then incubated with rabbit polyclonal antibody (anti-HER2) or a loading control, the mouse monoclonal antibody anti- $\alpha$-Tubulin (clone DM1A) (Cell Signaling Technology), at $4^{\circ} \mathrm{C}$ overnight. After extensive washing with tris-buffered saline tween, membranes were incubated with the secondary antibodies HRP-conjugated goat antirabbit and antimouse $\operatorname{IgG}_{1}$ for $1 \mathrm{~h}$ at room temperature. After final washing, proteins were visualized with a chemiluminescence detection system (Pierce) and subsequent exposure to the Kodak Molecular Imaging System. Protein band densities were analyzed using the spot density analysis software provided with the image station.

\section{Immunohistochemical Detection of HER2}

HER2 was analyzed immunohistochemically in paraffin sections using HercepTest, a Food and Drug Administration-approved assay for identification of tissue overexpressing p185 HER2 (K5205; Dako), in accordance with the manufacturer's protocol and scoring guidelines. The percentage of positive tumor cells was determined by experienced pathologists from the Pathology/ Histotechnology Laboratory of the National Cancer Institute at Frederick.

\section{Statistical Analysis}

Data are presented as mean $\pm \mathrm{SD}$. Statistical comparisons were made using the Student $t$ test. A $P$ value of less than 0.05 was considered significant.

\section{RESULTS}

\section{Association Between Tracer Uptake and HER2 Expression}

To assess the correlation between ${ }^{18} \mathrm{~F}-\mathrm{FBEM}-\mathrm{Z}_{\text {HER2:342 }}$ Affibody uptake and HER2 receptor density in vivo, we administered the tracer into mice bearing subcutaneous tumors with 5 levels of HER2 expression: BT474 (very 
high), MCF7/clone18 (high), MDA-MB-361 (medium), MCF7 (low), and MDA-MB-468 (negative). For all animals, 15-min static images were recorded $1 \mathrm{~h}$ after tracer injection. Afterward, tumor uptake was evaluated by semiquantitative analysis of PET images and confirmed by the biodistribution studies (Fig. 1). Good agreement was found between the results obtained by these 2 methods. These results also correlated well with the HER2 density corresponding to each particular tumor model, except for MCF7/ clone18, for which the specific uptake of ${ }^{18} \mathrm{~F}-\mathrm{FBEM}-$ $\mathrm{Z}_{\text {HER2:342 }}$ Affibody was much lower than we expected, based on ex vivo analysis of HER2 expression as measured in the cells and tissue lysates by ELISA (Table 1). Because this discrepancy could result from a different affinity of tracer binding to MCF7/clone18 cells, we checked ${ }^{18} \mathrm{~F}-$ FBEM-Z $Z_{H E R 2: 342}$ Affibody binding to MDA-MB-361 and MCF7/clone 18 cells in vitro by FACS analysis (Fig. 2). The results showed higher binding of Affibody molecules to MCF7/clone18 than to MDA-MB-361 cells. These data corresponded to the HER 2 expression measured in in vitro and ex vivo samples by ELISA and FACS, suggesting that the lower than expected observed accumulation of the tracer in MCF7/clone18 tumors is not caused by receptorbinding characteristics but most likely by differences in tumor microenvironment.

To check the feasibility of PET analysis as complementary to immunohistochemistry in routine clinical application, we also analyzed the same tissue samples by a standard immunostaining assay, HercepTest, according to score guidelines. Images of representative tumor cryosections stained for HER2 expression are shown in Figure 3. The receptor expression was found to be relatively homogeneous throughout the field of the sections. The immunohistochemistry scores were +3 for BT474, MCF7/clone18, and MDA-MB-361 cell lines and +1 for MCF7; MDAMB-468 cells were assessed as HER2-negative.

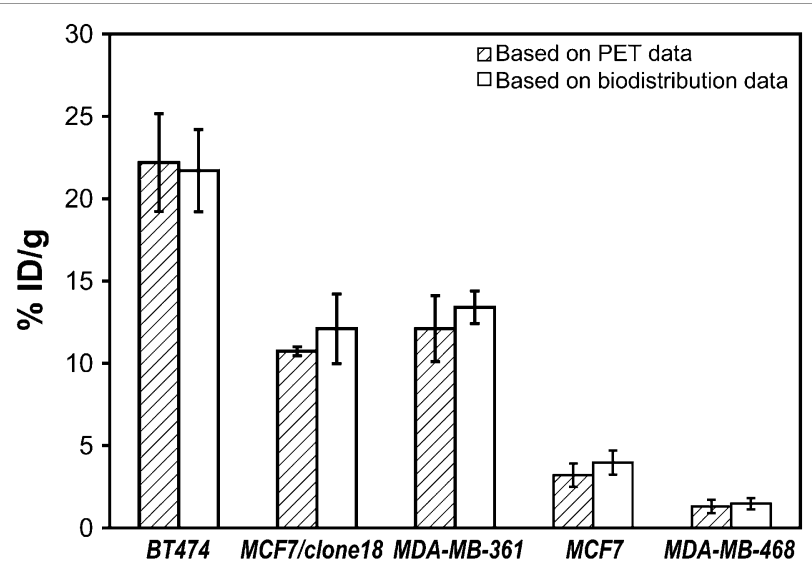

FIGURE 1. Comparison of quantification results obtained from PET region-of-interest analysis of tumors with different HER2 expression and direct $\gamma$-counter measurements, $1 \mathrm{~h}$ after ${ }^{18} \mathrm{~F}-\mathrm{FBEM}-\mathrm{Z}_{\text {HER2:342 }}$ Affibody injection $(n=4-6)$.
TABLE 1. Expression of HER2 in Breast Carcinoma Cells and Tissue Lysates

$\begin{array}{lcc}\text { Cell line/tissue } & \text { Cells }(\mathbf{n g} / \mathbf{m g}) & \text { Tissue }(\mathbf{n g} / \mathbf{m g}) \\ \text { BT474 } & 381 \pm 12.0 & 283 \pm 13.2 \\ \text { MCF7/clon18 } & 238 \pm 8.4 & 245 \pm 14.4 \\ \text { MDA-MB-361 } & 65 \pm 0.1 & 75 \pm 9.8 \\ \text { MCF7 } & 11 \pm 1.27 & 19 \pm 3.9 \\ \text { MDA-MB-468 } & 5 \pm 0.3 & 10 \pm 1.8\end{array}$

HER2 protein levels were measured by quantitative ELISA. Results reflect mean of duplicate measurements from 2 experiments in the case of cell studies and from 4-5 different tissue samples per group.

\section{Studies of Tracer Distribution}

Two-hour dynamic studies on mice bearing BT474 tumors were done to assess the kinetics of ${ }^{18} \mathrm{~F}-\mathrm{FBEM}-$ $\mathrm{Z}_{\text {HER2:342 Affibody accumulation in the tumors. The results }}$ showed that high-contrast PET image data can be collected $1 \mathrm{~h}$ after tracer injection. At that time, tumors were clearly distinguishable and tumor accumulation was the dominant feature. The biodistribution of the radioactivity $2 \mathrm{~h}$ after tracer injection was studied in mice bearing BT474, MCF7/ clone18, and MCF7 tumors through monitoring by PET.
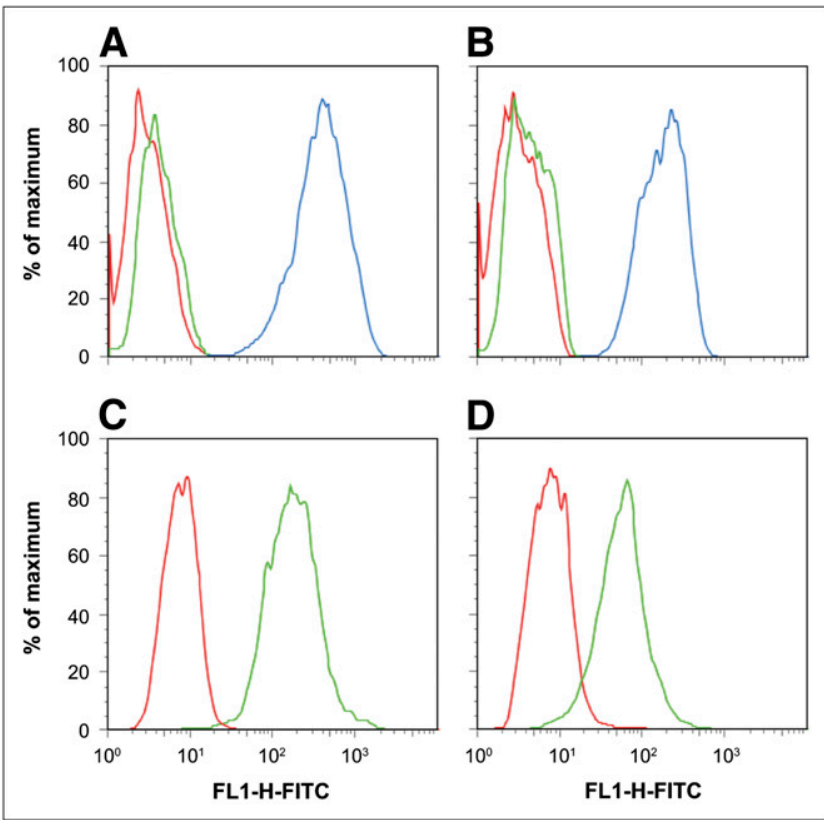

FIGURE 2. Flow cytometry histograms of HER2 receptor expression on MCF7/clone18 (A) and MDA-MB-361 (B) cell lines and of Affibody molecule binding to MCF7/clone18 (C) and MDA-MB-361 (D) cell lines. Each experiment was repeated 3 times; a representative histogram is shown for each cell line. Red curves in A and B correspond to nonstained control cells, green to isotypic control, and blue to HER2. In C and D, red curves correspond to isotypic control and green to Affibody binding. 

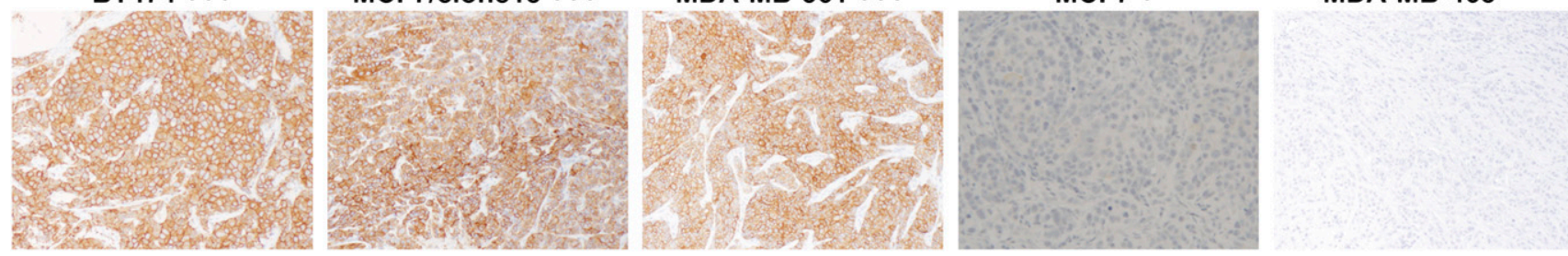

FIGURE 3. Immunohistochemical visualization of HER2 receptors in breast cancer tissues with different HER2 expression. HercepTest score for each cell line is shown.

The results agreed with previously published data (14). The biodistribution of the tracer as determined by $\gamma$-counter (Fig. 4) showed a high activity accumulation in BT474 tumors $(21.3 \pm 3.7 \% \mathrm{ID} / \mathrm{g})$ and in MCF7/clone18 tumors $(14.8 \pm 2.0 \% \mathrm{ID} / \mathrm{g})$ and was clearly lower $(P<0.004)$ in MCF7 tumors $(4.2 \pm 0.7 \% \mathrm{ID} / \mathrm{g})$. The highest normaltissue concentration of radioactivity was found in the kidneys. However, uptake of ${ }^{18} \mathrm{~F}-\mathrm{FBEM}-\mathrm{Z}_{\text {HER2:342 }}$ Affi- body was significantly lower in the kidneys than in the tumors of BT474 and MCF7/clone18 tumor-bearing mice and corresponded to $11.1 \pm 2.7 \% \mathrm{ID} / \mathrm{g}(P<0.02)$ and $5.8 \pm 2.1 \% \mathrm{ID} / \mathrm{g}(P<0.0002)$, respectively. Even for MCF7 tumor-bearing mice, the signal detected in the tumors and in the kidneys, although less pronounced because of the low expression of HER2 receptors on those tumors (Table1), was significantly different $(P<0.05)$.

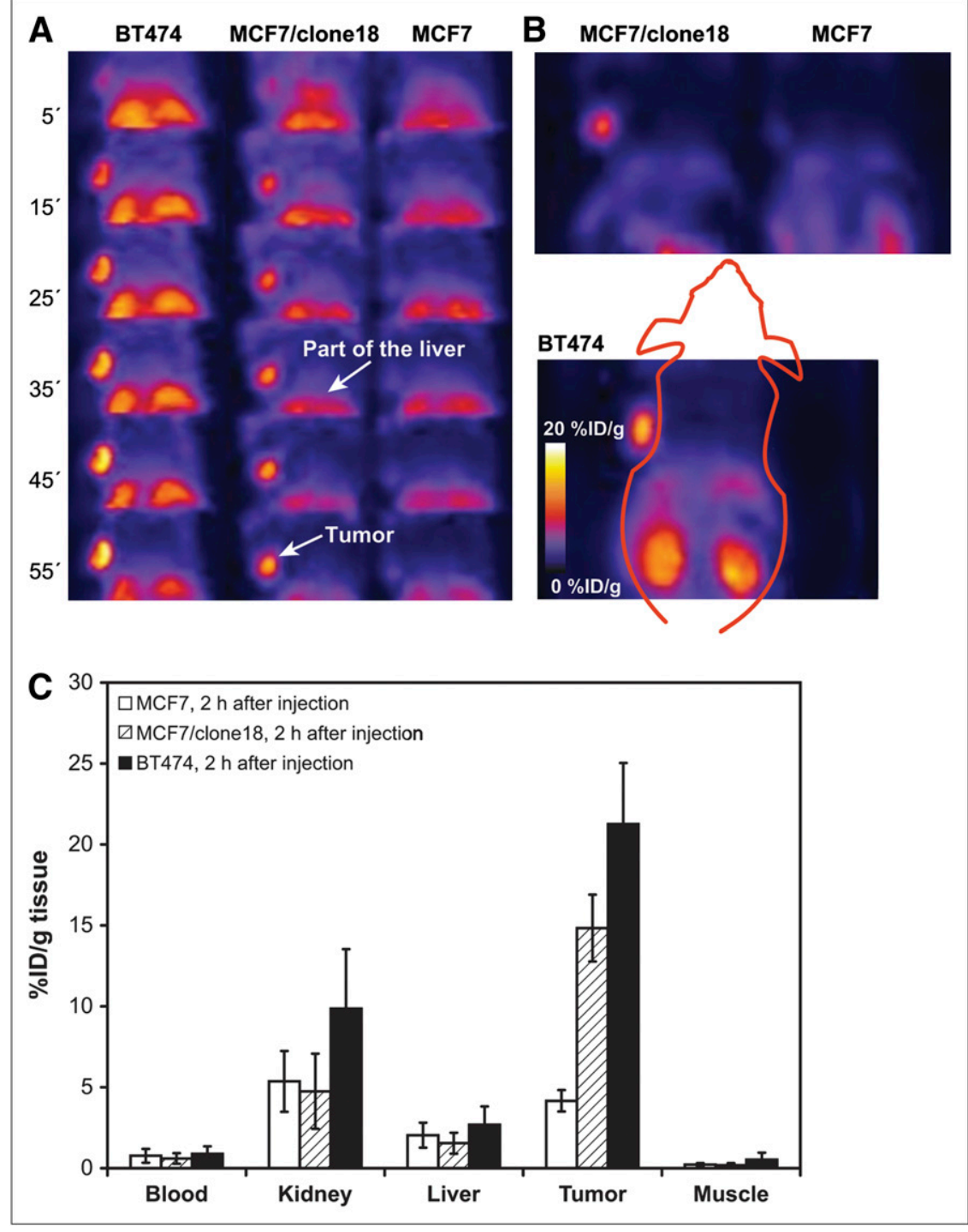

FIGURE 4. Representative coronal images obtained from 1-h dynamic study (A), coronal images obtained from whole-body study (B), and graph of biodistribution study (C) $2 \mathrm{~h}$ after ${ }^{18} \mathrm{~F}-\mathrm{FBEM}-\mathrm{Z}_{\text {HER2:342 }}$ Affibody molecule injection in nude mice carrying xenografts with different levels of HER2 expression $(n=6-7)$. 
Representative dynamic and whole-body coronal images of mice bearing xenografts with different HER2 expression are shown in Figure 4. Images with high tumor-to-background contrast were recorded for BT474 tumors, and it was still possible to detect MCF7 tumors with low HER2 expression.

\section{Monitoring Changes in HER2 Expression in Response to 17-DMAG}

The effect of therapeutic regimens on HER2 expression after 17-DMAG treatment was compared in mice bearing BT474 and MCF7/clone18 tumors.

Dynamic scanning was performed by acquiring a continuous series of 6 frames after tracer injection before and after 17-DMAG treatment. Next, 15-min static images were taken. In each case, a region of interest was placed around the tumor and muscle tissue and a time-activity curve or radioactivity concentration was calculated (Fig. 5). We decided to use maximal tumor uptake instead of mean tumor uptake, because the maximal tumor uptake measurement is free of user-dependent variation in defining regions of interest and is less susceptible to the effects of the necrosis that appears when tumors grow or respond to treatment during the monitoring time. Interestingly, based on image contrast, no particular differences in tumor uptake were observed before and after treatment (even though the animals received a high dose of the drug). However, the subsequent numeric analysis of recorded images clearly showed different ${ }^{18}$ F-FBEM-Z $Z_{\text {HER2:342 }}$ Affibody accumu- lations, confirming that the HER2 receptor expression had declined, compared with the pretreatment level. After 4 doses of 17-DMAG (40 mg/kg), tracer uptake was $71 \%$ lower $\left(P<4 \times 10^{-6}\right)$ in animals bearing BT474 tumors than in nontreated animals. The tumor response was less effective in mice bearing MCF7/clone18 tumors (only a $33 \%$ decrease, $P<0.002$ ). Final uptake of the tracer, determined by analysis of the images, was the same for both tumor models. To confirm these data, HER2 expression in the tissues extracted from the same tumors was also measured ex vivo by ELISA, immunoblot analysis, and an immunostaining assay using HercepTest. The numeric analysis is presented in Table 2, and the representative Western blots for BT474 and MCF7/clone18 tumor models are shown in Figure 5. In this case, we also obtained some unexpected results. According to the immunohistochemistry results, BT474 tumors expressing a high level of HER2 protein showed the same strong membrane and cytoplasmic staining corresponding to a score of $3+$ before and after treatment, whereas ex vivo analyses of BT474 tumor tissue lysates by Western blot and ELISA indicated that treatment with 17-DMAG resulted in significant downregulation of HER2 protein.

\section{DISCUSSION}

Molecular imaging of specific markers may provide information on both the expression of targeted molecules and the early assessment of response to a particular therapeutic intervention. Particularly PET, as a functional
FIGURE 5. Monitoring of effect of 17-DMAG on HER2 expression in mice bearing BT474 and MCF7/clone18 tumors. (A and C) Time-activity curves for tumor and muscle before and after 17-DMAG treatment in mice bearing BT474 tumors (A) and MCF7/clone18 tumors (C) (60-min dynamic studies; each frame, $10 \mathrm{~min}$ ). (B) Analysis of 15-min static images after dynamic studies. (D) Western blots of HER2 in BT474 and MCF7/clone18 tissue lysates before (control; left lane) and after (remaining lanes) treatment of mice with 17-DMAG. $\alpha$-Tubulin was used as loading marker.
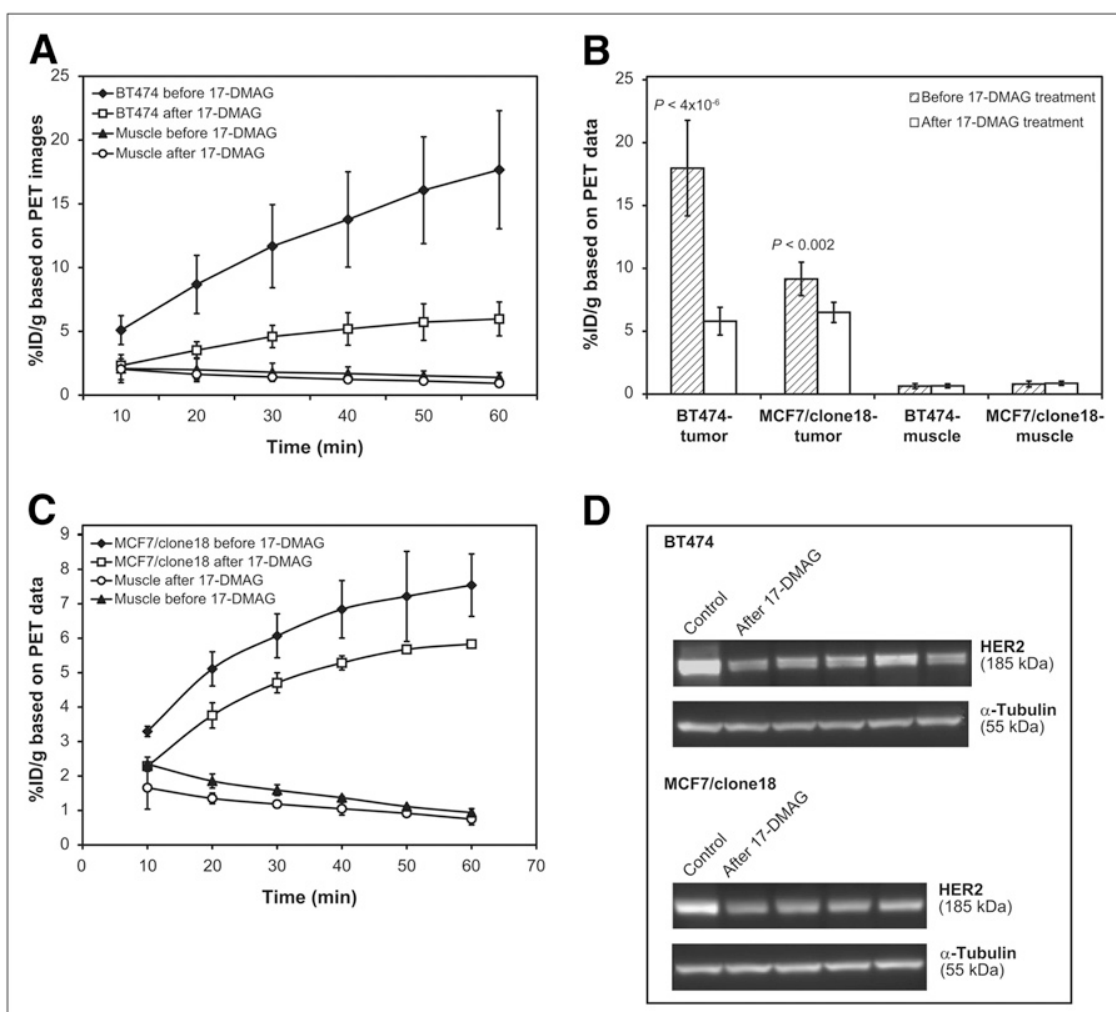


\begin{tabular}{|c|c|c|c|}
\hline Method & Timing & MCF7/clone18 & BT474 \\
\hline \multirow[t]{3}{*}{ PET (\%ID/g) } & Before & $9.16 \pm 1.33$ & $18.70 \pm 3.4$ \\
\hline & After & $6.50 \pm 0.83$ & $5.80 \pm 1.09$ \\
\hline & After/Before & $0.71 \pm 0.13$ & $0.33 \pm 0.08$ \\
\hline \multirow[t]{3}{*}{ Biodistribution (\%ID/g) } & Before & $12.00 \pm 1.56$ & $19.61 \pm 4.6$ \\
\hline & After & $8.57 \pm 1.31$ & $5.80 \pm 1.9$ \\
\hline & After/Before & $0.71 \pm 0.14$ & $0.30 \pm 0.11$ \\
\hline \multirow[t]{3}{*}{ ELISA (ng/mg) } & Before & $233.55 \pm 17.00$ & $354 \pm 12.7$ \\
\hline & After & $156.66 \pm 10.4$ & $111.63 \pm 11.80$ \\
\hline & After/Before & $0.67 \pm 0.07$ & $0.32 \pm 0.03$ \\
\hline \multirow[t]{3}{*}{ Western blot } & Before & 1 & 1 \\
\hline & After & $0.62 \pm 0.26$ & $0.28 \pm 0.07$ \\
\hline & After/Before & $0.62 \pm 0.26$ & $0.28 \pm 0.07$ \\
\hline
\end{tabular}

imaging technology, can provide rapid, reproducible, and noninvasive in vivo assessment of the receptor expression. Radioligands for in vivo imaging of HER2 by PET have been developed by several groups. Robinson et al. labeled a divalent antibody (C6.5) fragment with ${ }^{124}$ I and showed that it could be used for the imaging of HER2-positive tumors with a clinical PET/CT scanner (23). Garmestani et al. labeled intact trastuzumab with ${ }^{86} \mathrm{Y}$ or ${ }^{111} \mathrm{In}(24)$, whereas Olafsen et al. used trastuzumab to create a minibody and labeled it with ${ }^{64} \mathrm{Cu}(25)$. The resulting radioconjugate was successfully applied to image HER2 in tumor xenografts by small-animal PET. Smith-Jones et al. used an $\mathrm{F}\left(\mathrm{ab}^{\prime}\right)_{2}$ fragment of trastuzumab labeled with ${ }^{68} \mathrm{Ga}$ for PET (7). Their report presents the first attempt to monitor in vivo changes of HER2 expression after therapeutic intervention.

Many reports describing the application of Affibody molecules for imaging and therapy of HER2-positive tumors have been published by the Swedish group $(8,26-28)$. To our knowledge, we were the first to independently confirm the uniquely advantageous characteristics of these molecules as a HER2 targeting agent, especially for diagnostic application. By labeling the Affibody molecule containing a C-terminal cysteine $\left(\mathrm{Z}_{\text {HER2:342-Cys }}\right)$ with ${ }^{18} \mathrm{~F}$, we have created a new tracer that will allow the application of a well-established PET methodology to quantify HER2 expression in vivo. As described in our recent publication (14), the elimination half-life of about $36 \mathrm{~min}$ and high accumulation of the radioactivity in HER2-positive tumors, as compared with the background, facilitated the imaging studies. Our findings were then confirmed by Cheng et al., who also labeled Affibody molecules with ${ }^{18} \mathrm{~F}$ (15).

In this work, we have tested the sensitivity of our approach combining ${ }^{18} \mathrm{~F}$-labeled Affibody molecules and the PET technique to detect differences in HER2 expression levels and to monitor changes in HER2 expression after therapeutic intervention. To assess the correlation of signal observed by PET with receptor expression, we administered the new tracer to athymic nude mice bearing subcutaneous tumors with 5 levels of HER2 expression. The results showed that ${ }^{18} \mathrm{~F}-\mathrm{FBEM}-\mathrm{Z}_{\text {HER2:342 }}$ Affibody rapidly accumulated in HER2-positive tumors and was eliminated quickly from the blood and normal tissues, providing high tumor-to-blood and tumor-to-muscle ratios as soon as 20 min after injection. Notably, it was also possible to detect MCF7 tumors expressing a low level of HER2. The only other organs with higher accumulation than the tumors were the kidneys, indicating that clearance occurred exclusively via the renal tract. However, for monitoring breast tumors and even distant metastasis (except for adrenal and liver), reconstruction artifacts resulting in difficulties visualizing target will be negligible.

Semiquantitative PET analysis of tumors showed that the signal recorded by PET depended on the number of receptors expressed in those particular cell lines, as assessed by ex vivo methods. We found that PET data analysis corresponds well with the biodistribution results for each studied tumor model and depended on the HER2 expression, except for MCF7/clone18. The level of tracer binding in vivo in these tumors was comparable to the binding obtained in MDA-MB-361 xenografts (Fig. 1) despite the fact that the receptor expression in the latter was 3-fold lower (Table 1). In vitro FACS analysis of cell lysates (Fig. 2) showed that this divergence could not be explained by lower affinity of Affibody molecules to MCF7/clone18 cells. A similar discrepancy was observed by McLarty et al. (29). In these studies, the radioactivity uptake in MCF7/clone18 xenografts after injection of ${ }^{111}$ In-DTPA-trastuzumab was 2-fold lower than that observed in MDA-MB-361 cells, suggesting a nonlinear relationship between the imaging data and the actual concentration of the receptors. The authors also showed that the correlation can be disturbed by nonspecific binding. In our recent work, we investigated nonspecific binding of ${ }^{18} \mathrm{~F}-\mathrm{FBEM}-\mathrm{Z}_{\text {HER2:342 }}$ Affibody in both HER2- 
positive tumors after presaturation of the receptors with nonlabeled Affibody and HER2-negative tumor xenografts (14). Because our results showed the same level of binding in both cases, we assumed that nonspecific binding should be similar for other subcutaneous tumor xenografts and we decided to forego these experiments in the present work. It is likely that the lower than expected accumulation of radioactivity in MCF7/clone18 tumors could be due to vascular and microenvironmental abnormalities leading to inefficient penetration of the tracer. More rigorous PET studies allowing multicompartment kinetic analysis of the data might circumvent this problem.

Concluding that the signal from our tracer detected by PET in tumor tissue depends on different levels of HER2 expression, we moved on to evaluate its utility in monitoring changes in HER2 expression after treatment. It is known that 17-AAG and 17-DMAG cause downregulation of HER2 by interfering with the protective chaperone activity of Hsp90. Thus, to induce downregulation of HER2 in human breast cancer xenografts, we gave the animals 4 doses of 17-DMAG. This treatment, as applied to BT474 and MCF7/clone18 tumor-bearing mice, resulted in, respectively, $71 \%$ and $33 \%$ decreases in HER2 expression as observed by PET. Essentially the same reduction was observed by ex vivo assays, indicating that PET represents an attractive, noninvasive alternative for monitoring therapy effects on HER 2 expression and for optimizing the treatment schedule for individual patients. The results obtained for BT474 agreed well with the pharmacodynamic data previously reported by Smith-Jones et al. (7), who used 17-AAG and the same tumor model. Their study demonstrated an $80 \%$ reduction in HER2 expression in BT474 xenografts, which lasted approximately $36 \mathrm{~h}$, after which recovery in expression was observed. The same group has shown that downregulation of HER2, as measured by decreased uptake of $\mathrm{F}\left(\mathrm{ab}^{\prime}\right)_{2}$ fragments of trastuzumab, was more predictive than ${ }^{18} \mathrm{~F}$-FDG PET of a response to 17-AAG therapy in mice (30). Orlova et al. (8), using ${ }^{111} \mathrm{In}$ -

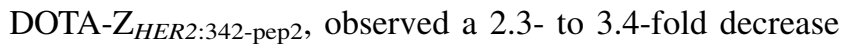
of signal from tumor volume on $\gamma$-camera images of mice bearing SKOV-3 tumors treated with 17-AGG.

We are not aware of any reports comparing the immunohistochemistry analysis with the more quantitative global ex vivo analysis of $H E R 2$ expression. Our studies indicated a significant discrepancy between the results obtained by HercepTest and ELISA or Western blot. For example, the pathology report based on guideline scores for HercepTest listed MDA-MB-361 tumors as 3+HER2-positive, even though our detailed ex vivo analysis showed a medium level of receptor expression, suggesting that the score should be $2+$. Similar disagreement between immunohistochemistry scoring of HER2 expression using ELISA and Western blot was observed in BT474 tumors treated with 17-DMAG. Strong membrane and cytoplasmic staining corresponding to a score of $3+$ was seen on both treated and nontreated tumors, whereas ex vivo analyses of the same tissues indicated that 17-DMAG caused a significant decrease of HER2 expression in the treated tumors. This inconsistency indicated that immunohistochemistry analysis has a subjective character that can be further distorted by the heterogeneity of HER2 expression in individual tumors.

Our approach using PET for in vivo assessment of HER2 expression may assist diagnosis of breast cancer and improve the outcome of HER2-targeted therapies. Immunohistochemistry and fluorescence in situ hybridization analyses are usually restricted to a limited tissue sample obtained at a single time. After initial diagnosis, follow-up biopsies are routinely not performed, and currently, there is no means to obtain information on how long it takes a therapeutic agent such as Herceptin to reach the target, how effective it is, and how long its effect lasts. Moreover, the high level of discordance between these 2 methods has been documented in several studies $(31,32)$. Discordance rates may be as high as $20 \%$ when HER2 testing is performed in low volume and at local laboratories (33). As we have shown in this work, PET using ${ }^{18}$ F-labeled Affibody molecules was sufficiently sensitive to detect a 2- to 3-fold decrease in HER2 expression, whereas immunohistochemistry not only was less sensitive but also misclassified some tumors with intermediate levels of $H E R 2$ as highly overexpressing. Such inaccurate estimates of HER2 expression by immunohistochemistry analysis might lead to misdiagnosis and severe clinical consequences. Therefore, PET may provide a considerable advantage and become an attractive alternative for assessing HER2 expression quantitatively, objectively, and noninvasively, allowing several scans to be performed over the course of therapy.

\section{CONCLUSION}

Our results indicate that $H E R 2$ expression in vivo and its possible changes in response to therapeutic interventions can be monitored by PET using ${ }^{18} \mathrm{~F}-\mathrm{FBEM}-\mathrm{Z}_{\text {HER2:342 }}$ Affibody. This approach represents an attractive, noninvasive alternative for monitoring therapeutic effects on HER2 expression and for optimizing the treatment schedule for individual patients.

\section{ACKNOWLEDGMENTS}

We appreciate the support of experts from Affibody $A B$ and SAIC Frederick, Inc.; the technical assistance of Yesenia Rodriguez and Aliesia Holly; and constructive discussions with Lucia Martiniova and one of the creators of ATLAS, Jürgen Seidel. The content of this publication does not necessarily reflect the views or policies of the Department of Health and Human Services, nor does mention of trade names, commercial products, or organizations imply endorsement by the U.S. government. This research was supported in part by the Center for Cancer Research (an intramural research program of the National Cancer Institute), the National Institute of Biomedical Imaging and 
Bioengineering, a Breast Cancer Research Stamp Fund awarded through competitive peer review, and federal funds from the National Cancer Institute, National Institutes of Health (contracts N01-CO-12400 and N01-CO-12401).

\section{REFERENCES}

1. Meric-Bernstam F, Hung MC. Advances in targeting human epidermal growth factor receptor-2 signaling for cancer therapy. Clin Cancer Res. 2006;12:63266330.

2. Rabindran SK. Antitumor activity of HER-2 inhibitors. Cancer Lett. 2005; 227:9-23.

3. Friedlander E, Barok M, Szollosi J, Vereb G. ErbB-directed immunotherapy: antibodies in current practice and promising new agents. Immunol Lett. 2008; 116:126-140.

4. Whitesell L, Lindquist SL. HSP90 and the chaperoning of cancer. Nat Rev Cancer. 2005;5:761-772.

5. Uehara Y, Hori M, Takeuchi T, Umezawa H. Phenotypic change from transformed to normal induced by benzoquinonoid ansamycins accompanies inactivation of p60src in rat kidney cells infected with Rous sarcoma virus. Mol Cell Biol. 1986;6:2198-2206.

6. Hollingshead M, Alley M, Burger AM, et al. In vivo antitumor efficacy of 17DMAG (17-dimethylaminoethylamino-17-demethoxygeldanamycin hydrochloride), a water-soluble geldanamycin derivative. Cancer Chemother Pharmacol. 2005;56:115-125.

7. Smith-Jones PM, Solit DB, Akhurst T, Afroze F, Rosen N, Larson SM. Imaging the pharmacodynamics of HER2 degradation in response to Hsp90 inhibitors. Nat Biotechnol. 2004;22:701-706.

8. Orlova A, Tolmachev V, Pehrson R, et al. Synthetic affibody molecules: a novel class of affinity ligands for molecular imaging of HER2-expressing malignant tumors. Cancer Res. 2007;67:2178-2186.

9. Neckers L. Heat shock protein 90: the cancer chaperone. J Biosci. 2007;32: 517-530.

10. Dowsett M, Hanna WM, Kockx M, et al. Standardization of HER2 testing: results of an international proficiency-testing ring study. Mod Pathol. 2007; 20:584-591.

11. Tsuda H. HER-2 (c-erbB-2) test update: present status and problems. Breast Cancer. 2006;13:236-248.

12. Imai K, Takaoka A. Comparing antibody and small-molecule therapies for cancer. Nat Rev Cancer. 2006;6:714-727.

13. Jain M, Kamal N, Batra SK. Engineering antibodies for clinical applications. Trends Biotechnol. 2007;25:307-316.

14. Kramer-Marek G, Kiesewetter DO, Martiniova L, et al. [ ${ }^{18}$ F]FBEMZ(HER2:342)-Affibody molecule-a new molecular tracer for in vivo monitoring of HER2 expression by positron emission tomography. Eur J Nucl Med Mol Imaging. 2008;35:1008-1018.

15. Cheng Z, De Jesus OP, Namavari M, et al. Small-animal PET imaging of human epidermal growth factor receptor type 2 expression with site-specific ${ }^{18} \mathrm{~F}$-labeled protein scaffold molecules. J Nucl Med. 2008;49:804-813.

16. Nordberg E, Orlova A, Friedman M, et al. In vivo and in vitro uptake of ${ }^{111} \mathrm{In}$, delivered with the affibody molecule (ZEGFR:955)2, in EGFR expressing tumour cells. Oncol Rep. 2008;19:853-857.
17. Ekblad T, Tran T, Orlova A, et al. Development and preclinical characterisation of ${ }^{99 \mathrm{~m}}$ Tc-labelled Affibody molecules with reduced renal uptake. Eur J Nucl Med Mol Imaging. 2008;35:2245-2255.

18. Tran T, Engfeldt T, Orlova A, et al. ${ }^{99 \mathrm{~m} T c-m a E E E-Z(H E R 2: 342), ~ a n ~ A f f i b o d y ~}$ molecule-based tracer for the detection of HER2 expression in malignant tumors. Bioconjug Chem. 2007;18:1956-1964.

19. Tolmachev V, Nilsson FY, Widstrom C, et al. ${ }^{111}$ In-benzyl-DTPA-ZHER2:342, an affibody-based conjugate for in vivo imaging of HER2 expression in malignant tumors. J Nucl Med. 2006;47:846-853.

20. Kiesewetter DO, Kramer-Marek G, Ma Y, Capala J. Radiolabelling of Her2 specyfic affibody molecule with F-18. J Fluor Chem. 2008;129:799-806.

21. Guide for the Care and Use of Laboratory Animals. Washington, DC: National Academy Press; 1996.

22. Seidel J. Resolution uniformity and sensitivity of the NIH atlas small animal PET scanner: comparison to simulated LSO scanners without depth-of-interaction capability. IEEE Trans Nucl Sci. 2003;50:1347-1357.

23. Robinson MK, Doss M, Shaller C, et al. Quantitative immuno-positron emission tomography imaging of HER2-positive tumor xenografts with an iodine-124 labeled anti-HER2 diabody. Cancer Res. 2005;65:1471-1478.

24. Garmestani K, Milenic DE, Plascjak PS, Brechbiel MW. A new and convenient method for purification of ${ }^{86} \mathrm{Y}$ using a $\mathrm{Sr}$ (II) selective resin and comparison of biodistribution of ${ }^{86} \mathrm{Y}$ and ${ }^{111}$ In labeled Herceptin. Nucl Med Biol. 2002;29:599606.

25. Olafsen $\mathrm{T}$ Kenanova VE Sundaresan $\mathrm{G}$, et al. Optimizing radiolabeled engineered anti-p185HER2 antibody fragments for in vivo imaging. Cancer Res. 2005;65:5907-5916.

26. Orlova A, Feldwisch J, Abrahmsen L, Tolmachev V. Update: affibody molecules for molecular imaging and therapy for cancer. Cancer Biother Radiopharm. 2007;22:573-584.

27. Steffen AC, Orlova A, Wikman M, et al. Affibody-mediated tumour targeting of HER-2 expressing xenografts in mice. Eur J Nucl Med Mol Imaging. 2006;33: 631-638.

28. Mume E, Orlova A, Larsson B, et al. Evaluation of ((4-hydroxyphenyl)ethyl)maleimide for site-specific radiobromination of anti-HER2 affibody. Bioconjug Chem. 2005;16:1547-1555.

29. McLarty K, Cornelissen B, Scollard DA, et al. Associations between the uptake of ${ }^{111}$ In-DTPA-trastuzumab, HER2 density and response to trastuzumab (Herceptin) in athymic mice bearing subcutaneous human tumour xenografts. Eur J Nucl Med Mol Imaging. 2009;36:81-93.

30. Smith-Jones PM, Solit D, Afroze F, Rosen N, Larson SM. Early tumor response to Hsp90 therapy using HER2 PET: comparison with ${ }^{18}$ F-FDG PET. J Nucl Med. 2006;47:793-796.

31. Todorovic-Rakovic N, Jovanovic D, Neskovic-Konstantinovic Z, NikolicVukosavljevic D. Comparison between immunohistochemistry and chromogenic in situ hybridization in assessing HER-2 status in breast cancer. Pathol Int. 2005;55:318-323.

32. Gokhale S, Gatalica Z, Mohammad A, Rampy AI, Velagaleti Gopalrao VN. FISH for HER-2/neu in breast cancer: standardization makes the difference! Indian J Cancer. 2004;41:152-158.

33. Moeder CB, Giltnane JM, Harigopal M, et al. Quantitative justification of the change from $10 \%$ to $30 \%$ for human epidermal growth factor receptor 2 scoring in the American Society of Clinical Oncology/College of American Pathologists guidelines: tumor heterogeneity in breast cancer and its implications for tissue microarray based assessment of outcome. J Clin Oncol. 2007;25:5418-5425. 